\title{
The depth and selectivity of suppression in binocular rivalry
}

\author{
VINCENT A. NGUYEN and ALAN W. FREEMAN \\ University of Sydney, Lidcombe, Australia \\ and \\ PETER WENDEROTH \\ Macquarie University, North Ryde, Australia
}

\begin{abstract}
Binocular rivalry occurs when the two eyes are presented with incompatible stimuli and the perceived image alternates between the two stimuli. The aim of this study was to find out whether the periodic perceptual loss of a monocular stimulus during binocular rivalry is mirrored by a comparable loss of contrast sensitivity. We presented brief test stimuli to one eye while its conditioning stimulus was dominant or suppressed. The test stimuli were varied widely across four stimulus domainsnamely, the relative stimulation of medium- and long-wavelength-sensitive cones, duration, spatial frequency, and grating orientation. The result in each case was the same. Suppression depended slightly or not at all on the type of test stimulus, and contrast sensitivity during suppression was around $64 \%$ of that during dominance. The effect of suppression on sensitivity is therefore very weak, relative to its effect on the perceived image. Furthermore, suppression was largely independent of the similarity between the conditioning and the test stimuli, indicating that our results are better explained by eye suppression than by stimulus suppression. A model is presented to account for the small, monocular sensitivity loss during suppression: It assumes that test detection precedes conditioning stimulus perception in the visual pathway.
\end{abstract}

Binocular rivalry is easily demonstrated by presenting a small area of grating to each eye, with one eye's grating orthogonal to the other. The viewer typically sees only one grating or the other, with the dominant percept swapping from one grating to the other every few seconds. This phenomenon has fascinated students of the visual system for hundreds of years (DuTour, 1760), because it dramatically alters the perceived image in the absence of any change in the visual stimulus. Given the marked change in perception during rivalry, it makes sense to ask whether visual sensitivity is similarly altered. A number of studies have looked at this question by briefly presenting test stimuli to one eye during its dominance phase, when the conditioning stimulus to that eye is most visible, and during its suppression phase, when the conditioning stimulus is least visible. One of the earliest studies (Wales \& Fox, 1970) found that the sensitivity for detecting a test stimulus was a factor of three lower during the suppression phase than during the dominance phase. Later stud-

Portions of this work have been previously published in Nguyen and Freeman (1997) and Nguyen, Freeman, Wenderoth, and Heard (1998). This project was supported by a National Health and Medical Research Council Equipment Grant. We thank Robert Heard for help with the statistical analysis of the data. Correspondence concerning this article should be addressed to A. W. Freeman, School of Biomedical Sciences, University of Sydney, P. O. Box 170, Lidcombe, NSW 1825, Australia (e-mail: a.freeman@cchs.usyd.edu.au). ies (Blake \& Camisa, 1979; Makous \& Sanders, 1978) have found sensitivity losses of a similar order.

This result leads to a major puzzle. Rivalry can prevent the perception of a conditioning stimulus, and yet it reduces visual sensitivity by only a small amount. The discrepancy is more obvious when stated in terms of contrast. To prevent the detection of the (typically high-contrast) conditioning stimulus under nonrivalry conditions would require reducing its contrast by a factor of around 100. Yet when the sensitivity of the suppressed eye is tested during rivalry, the loss of effective contrast is closer to threefold. How can this discrepancy be explained? One possibility is that the suppression that operates during binocular rivalry is selective for certain stimulus features, that the features most heavily suppressed are those most important for perception, and that less suppressed features contribute to visual sensitivity.

Counting against this hypothesis are a number of studies showing that when a conditioning stimulus is suppressed, alterations in its temporal or spatial makeup do not improve its visibility (Blake \& Fox, 1974b; Blake, Westendorf, \& Overton, 1980; Fox \& Check, 1968; Wales $\&$ Fox, 1970). Fox and Check (1968) induced rivalry and then rotated or translated the suppressed conditioning stimulus. Stimulus motion did not terminate the suppression period, so long as the motion was slow. Blake and Fox (1974b) changed the spatial frequency or orientation of a suppressed grating and likewise found that altering 
the conditioning stimulus did not terminate the suppression period. These studies lead to the conclusion that the suppression process indiscriminately reduces the visibility of a wide variety of stimuli.

There is other evidence, however, suggesting that suppression is selective. Logothetis, Leopold, and Sheinberg (1996) induced binocular rivalry with orthogonal gratings and then swapped the gratings between eyes every third of a second. The interval during which a grating of fixed orientation was continuously perceived spanned $2 \mathrm{sec}$ or more, despite the fact that the grating was swapped between eyes several times during that period. Lee and Blake (1999) have carefully defined the conditions under which this observation occurs. These results lead to the possibility that suppression is selectively targeted at a stimulus feature, regardless of the eye to which that feature is presented. Logothetis et al. refer to this as stimulus suppression, as opposed to eye suppression.

Kovács, Papathomas, Yang, and Fehér (1996) also provided evidence for stimulus suppression. They presented an array of spots to one eye, with some spots green and the remainder red. A spatially identical array of spots was presented to the fellow eye, but with green spots swapped for red, and red for green. When the subjects fused the two arrays, they saw spots of a single color much more often than would be predicted on the basis of rivalry between the two eyes. Spots of the other color were suppressed, even though some were presented to one eye and the remainder to the fellow eye.

Clear evidence of selectivity in the suppression process is obtained when the chromatic properties of the stimulus are altered (Ooi \& Loop, 1994; E. L. Smith, Levi, Harwerth, \& White, 1982). E. L. Smith et al. presented orthogonal gratings to the two eyes, resulting in binocular rivalry, and used a flashed test stimulus to determine the chromatic sensitivity of one eye during both its dominant and its suppressed phases. Sensitivity during dominance peaked at three separate stimulus wavelengths, indicating that the test stimulus was being detected by three channels differing in their chromatic properties. During suppression, however, sensitivity formed a onepeaked function of wavelength that was well fitted by the photopic luminosity function. E. L. Smith et al. concluded that signals in chromatic visual channels are reduced more than those in luminance channels during suppression.

E. L. Smith et al.'s (1982) result suggests a possible reason for the discrepancy between large perceptual losses and small sensitivity losses during binocular rivalry. Perhaps the perceptual loss during rivalry is due to the reduced signal in chromatic channels, whereas the detection of a test stimulus during suppression is mediated by the relatively unaffected luminance channels. We tested this idea by delivering chromatically modulated stimuli during both the dominance and the suppression phases of rivalry. Chromatic and luminance channels were tested independently by varying the ratio in which the test stimulus activated long- and medium-wavelength-sensitive cones. Another possibility is that suppression acts selec- tively on temporal or spatial stimulus domains that have not been explicitly tested in previous work. We have therefore varied the duration and spatial frequency of the test stimulus over a wide range, to see whether a portion of the range is specifically affected by suppression. Finally, we determined whether loss of sensitivity for a test stimulus is due to eye suppression or stimulus suppression by altering the orientation of the test so that it was either similar or dissimilar spatially to one of the conditioning stimuli. Stimulus suppression predicts greater suppression depth when the test stimulus is similar to the suppressed conditioning stimulus.

\section{METHOD}

\section{Subjects}

Ten subjects were tested. The age range was $21-35$ years, and all the subjects had visual acuities of $6 / 6$ or better in each eye. Stereopsis was measured with the TNO test and showed that the binocular disparity threshold was $2 \mathrm{~min}$ arc or better. All the subjects gave written consent to their participation once the methodology had been explained to them. Apart from one of the authors (V.A.N.), the subjects were unaware of the purpose and results of the experiments.

\section{Stimulus}

The two eyes were presented with differing static conditioning stimuli to produce binocular rivalry, and a test stimulus was then presented to one eye to measure its contrast sensitivity. When visual stimuli delivered to the two eyes differ over a large area, the perception is of a patchwork in which the stimulus delivered to one eye is perceived in some patches and the other stimulus dominates in the remaining patches. We ensured that suppression was largely restricted to one eye's stimulus at a time by using binocularly incompatible conditioning stimuli with a diameter of only $1^{\circ}$ (Blake, O'Shea, \& Mueller, 1992). Furthermore, the test stimuli were kept brief to make sure that they did not overlap the interval in which suppression was transferring from one eye's stimulus to the other.

Visual stimuli were delivered on a computer monitor (Mitsubishi HL7955 or NEC 4FG) driven by a high-resolution video card (VSG $2 / 2$, Cambridge Research Systems). The monitor's interpixel spacing was $0.2 \mathrm{~min}$ arc, and its optical distance from the eye was $6 \mathrm{~m}$, except in Experiment 2, where it was $5 \mathrm{~m}$. The monitor's frame rate was $100 \mathrm{~Hz}$ in Experiment 1, $70 \mathrm{~Hz}$ in Experiment 2, and $120 \mathrm{~Hz}$ in Experiments 3 and 4. Stimuli for the left eye were presented on the left half of the monitor screen, and stimuli for the right eye on the right half. A septum placed in front of the screen and front-surfaced mirrors placed along the optical path kept the left-eye and the righteye views separate. The mirror in front of each eye belonged to a stereoscope, which the subject adjusted to bring the two views into alignment. The subject used chin and forehead rests to reduce head movement.

The conditioning stimulus to each eye subtended $2.2^{\circ}$ horizontally and $3.5^{\circ}$ vertically. It included a centered annulus to aid binocular fusion and Nonius markers around the edge to indicate the fusion status, as is shown in Figure 1A. The annulus had inner and outer radii of $1^{\circ}$ and $1.12^{\circ}$, respectively, the Nonius markers were squares $0.2^{\circ}$ on a side, and both the annulus and the markers were dark. The bright region outside the annulus was spatially uniform, with a luminance of $100 \mathrm{~cd} \mathrm{~m}^{-2}$. The spatial pattern within the annulus was a 3 cycles deg $^{-1}$ grating for both eyes, with one grating oriented orthogonally to the other. The gratings had a contrast of $50 \%$ and a mean luminance of $66 \mathrm{~cd} \mathrm{~m}^{-2}$ (in Experiments 1 and 2) or $50 \mathrm{~cd} \mathrm{~m}^{-2}$ (in Experiments 3 and 4). All the experiments were performed in a dark room so that the only significant source of light came from the monitor screen. 
A

\section{Time Left eye view Right eye view}

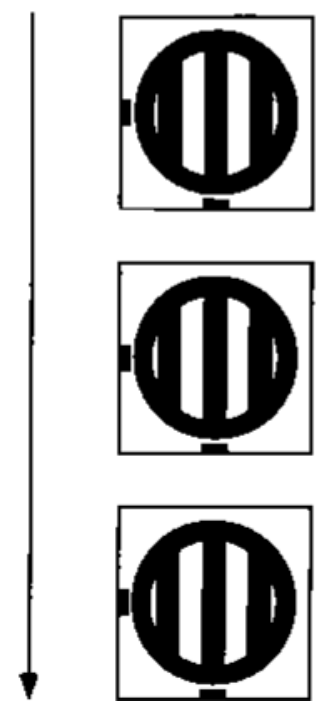

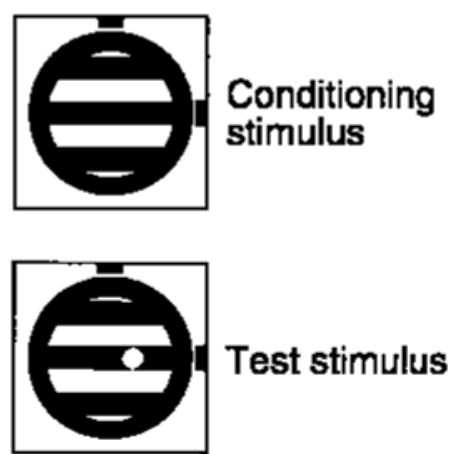

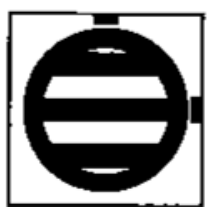

Subject makes judgement

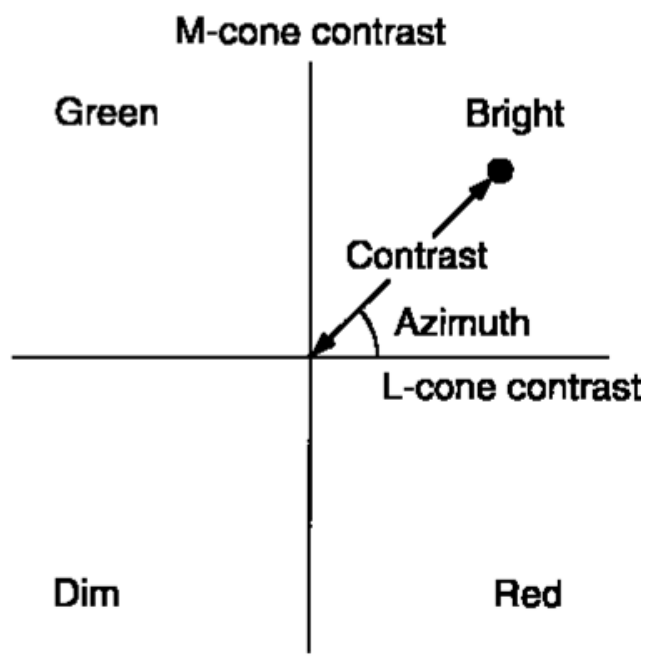

Figure 1. Stimulus used to test sensitivity to briefly flashed targets during binocular rivalry. (A) Spatial and temporal design of the experiment. Orthogonal, square-wave gratings were used to induce rivalry. The gratings had a spatial frequency of $3 \mathrm{cycles} \mathrm{deg}^{-1} \mathrm{and}$ a contrast of $50 \%$. Surrounding each grating were an annulus to aid binocular fusion and Nonius lines to monitor fusion status. When the grating viewed by the right eye was dominant or suppressed, depending on the type of trial, the subject triggered a test stimulus. The test was a uniform spot contained within the central dark bar of the right eye's grating. Test contrast was calculated relative to the dark bar. The test stimulus was displaced one diameter to the left or right of midline in the right eye's view; only the latter case is shown. The subject's task was to signal on which side the test stimulus appeared. (B) Color space used to specify test stimulus chromaticity. The horizontal and vertical axes show contrast for the long- (L-) and medium- (M-) wavelength-sensitive cones, respectively. The background stimulus produces zero contrast and therefore plots at the origin. The appearances of stimuli represented by each quadrant are indicated (for example, "Bright" in the upper right quadrant). A point in this space is defined by the vector connecting it to the origin and, therefore, by the length of the vector (contrast) and its angle with the horizontal (azimuth).

Test stimuli were delivered to the right eye. Test onset and offset were step functions of time; test duration was $100 \mathrm{msec}$ for Experiments 1, 3, and 4 and was variable in Experiment 2. For experiments apart from the first, test contrast was set equal to the luminance increment from the test divided by the background luminance. In Experiment 1, the test stimulus differed from the background in both chromaticity and luminance; the difference was defined by the cone contrasts of the test. Cone contrast was determined as follows. First, cone energy absorption was calculated by measuring the spectral emission of the stimulator screen with a spectrophotometer (Photo Research PR-650), weighting the emission with the spectral sensitivity of the relevant cone type (V. C. Smith \& Pokorny, 1975), and integrating over wavelength. Cone contrast was then set equal to the increment in cone energy absorption from the test stimulus divided by the absorption from the background. Contrast for the short-wavelength-sensitive (S-) cones was set equal to zero so that only the contrasts of the medium- and long-wavelength-sensitive cones (M- and L-cones, respectively) were free to vary. Test stimulus contrast was checked by measuring test chromaticity and luminance with the spectrophotometer. Measured contrast deviated from a linear function of contrast setting by at most $3 \%$.

Figure 1B shows the coordinate system by which the chromatic content of the test stimulus was varied. The horizontal axis gives Lcone contrast, the vertical axis gives M-cone contrast, and a stimulus with zero cone contrast is identical to the background so that the origin represents the background. A test stimulus with a higher luminance than the background increases both L- and M-cone responses and therefore appears in the upper right quadrant. A satu- rated green test stimulus decreases L- and increases M-cone responses, thereby appearing in the upper left quadrant. Similarly, low luminance and red stimuli appear in the lower left and right quadrants, respectively. An arbitrary test stimulus can be represented by the length of the line joining it to the origin and the angle between this line and the horizontal axis. These two quantities will be termed contrast and azimuth, respectively.

\section{Psychophysical Method}

The subject waited until one eye's conditioning stimulus was completely dominant, so that there was no trace of the incompatible conditioning stimulus delivered to the other eye. The subject then triggered a test stimulus, which was delivered at either of two locations. The location to which it was assigned varied randomly from trial to trial, and the subject's task was to signal in which location it appeared. Incorrect choices were indicated with a short tone. Stimulus contrast was varied with an up-down staircase rule: Contrast was increased by a multiplicative factor of 1.25 after each incorrect response and decreased by the same factor after three consecutive correct responses. This process was continued until four down- and three up-staircases were completed. A Weibull function (Weibull, 1951) was fitted to the resulting psychometric curve, and the contrast threshold was set at that point on the function for which the fraction of correct responses was .79. Contrast sensitivity was calculated as the reciprocal of contrast threshold. At least two staircases were interleaved in a run so that the subject could not predict what stimulus would appear next. For each stimulus type, the final contrast sensitivity was the mean across three or four runs. 

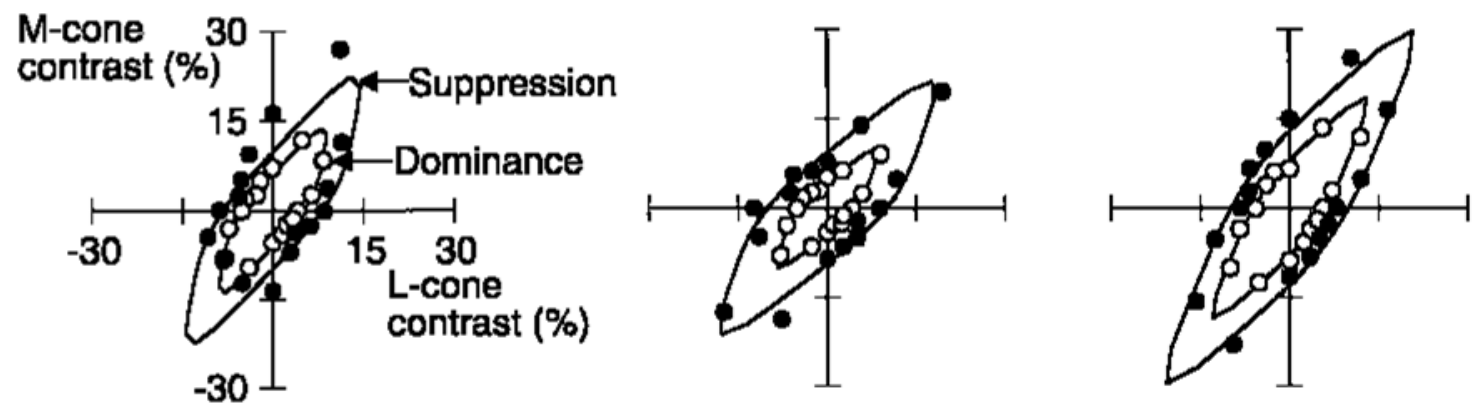

B
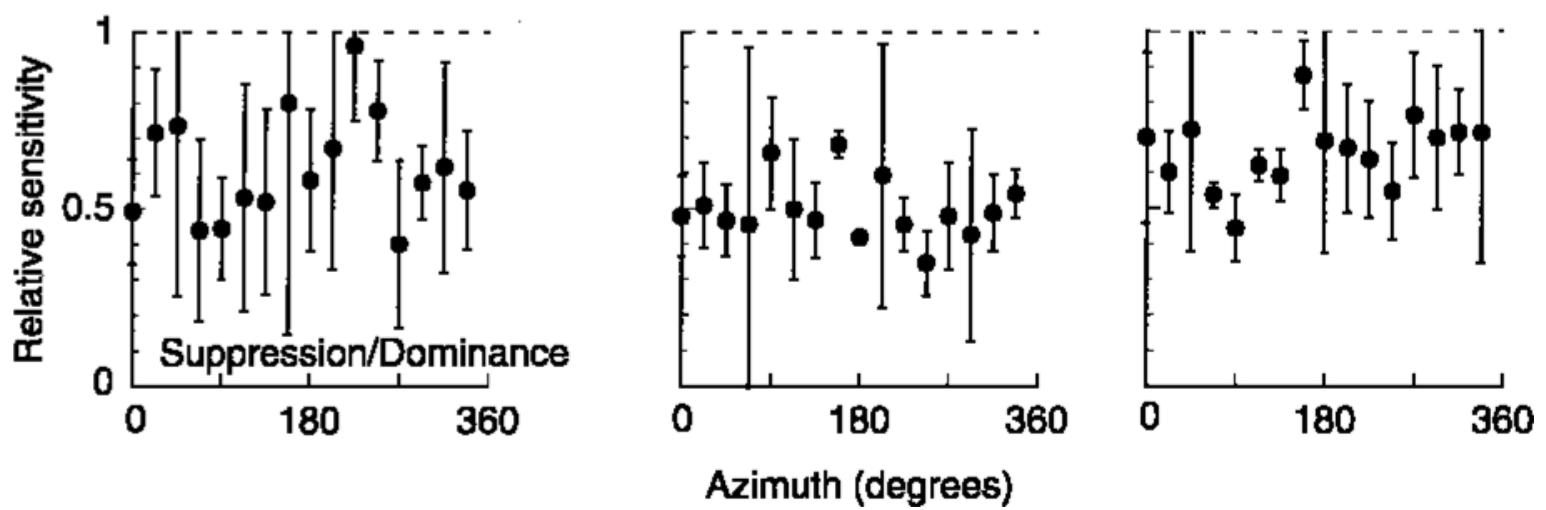

Figure 2. Effect of binocular rivalry suppression on color vision. (A) Contrast thresholds for 3 subjects stimulated with 16 combinations of $L$ - and $M$-cone contrast. Thresholds obtained during the dominance and suppression phases of rivalry are shown with open and filled circles, respectively. The contour through the open circles shows the best fit for a probability summation model (Equation A1). The contour was expanded uniformly to fit the closed circles. (B) Suppression depth as a function of polar angle in the color space. Suppression depth was calculated by dividing contrast threshold during dominance by that during suppression. The dashed line indicates an absence of suppression, and each error bar indicates the $95 \%$ confidence interval.

\section{RESULTS}

\section{Experiment 1: Chromatic}

\section{Selectivity of Suppression}

In this experiment, we tested whether suppression strength depends on the chromatic content of a test stimulus. Figure 1A shows the design of the experiment. Binocular rivalry was induced with orthogonal gratings, and the test stimulus appeared when triggered by the subject. The test was a spot of $10 \mathrm{~min}$ arc diameter that was superimposed on a dark bar of the grating presented to the right eye. The test was displaced to the left or right of center of the right eye's view, and the subject's task was to signal whether the test was displaced left or right. The duration of the test stimulus was fixed at $100 \mathrm{msec}$. The chromaticity of the test stimulus is given by its azimuth, which indicates the relative activation of $\mathrm{L}$ - and $\mathrm{M}$-cones, as shown in Figure 1B.

Figure 2A gives the contrast thresholds for 3 subjects. The open circles, representing the dominance phase for the tested eye, were obtained by presenting stimuli at 16 azimuths evenly distributed around the origin. Contrast thresholds are greatest in the upper right and lower left quadrants, indicating that luminance changes are more difficult to detect than chromaticity changes. This observation matches well with previously published work (Chaparro, Stromeyer, Huang, Kronauer, \& Eskew, 1993; Cole, Hine, \& McIlhagga, 1993). The filled circles give contrast threshold during suppression. They form a contour lying outside the contour obtained during dominance, indicating that contrast threshold increases during suppression for all directions in this color space.

The graphs in Figure 2B show whether suppression depth is greater at some azimuths than at others. These data were obtained by dividing contrast threshold during dominance by that during suppression, and the loss owing to suppression is represented by the displacement of an ordinate below the dashed line. If chromatic channels were more suppressed than luminance channels, as was suggested by E. L. Smith et al. (1982), suppression depth would be greater at azimuths close to $135^{\circ}$ and $315^{\circ}$, where the test stimulus is isoluminant with the background. There is a suggestion of such a trend in the data from subject D.L., but no such trend is evident for the other subjects.

An analysis of variance (ANOVA) was performed by pooling sensitivities across subjects. The interaction term 

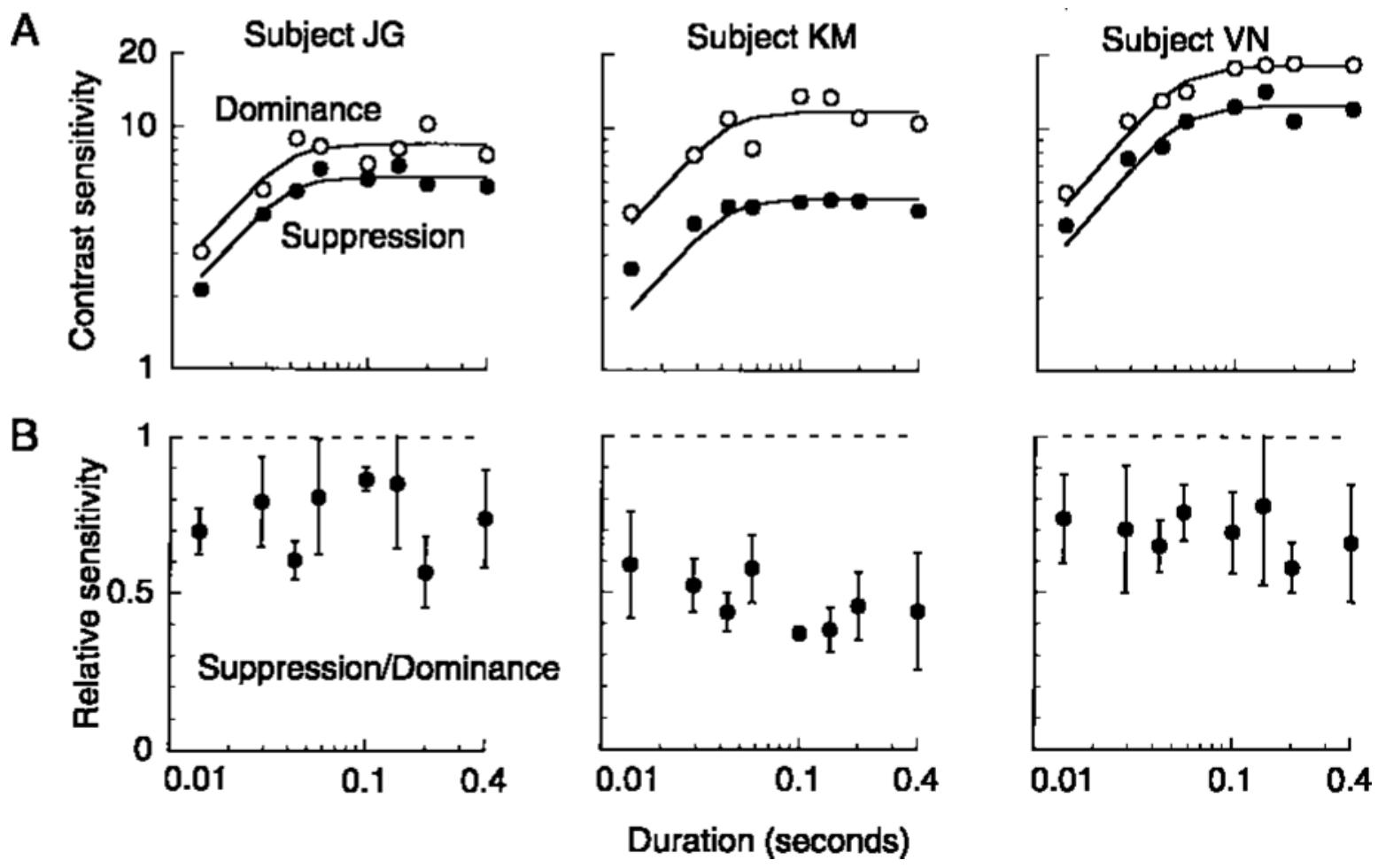

Figure 3. Dependence of binocular rivalry suppression on test stimulus duration. (A) Contrast sensitivity as a function of test stimulus duration for 3 subjects. Sensitivities during the dominance and suppression phases of binocular rivalry are shown by open and filled circles, respectively. The line fitted to the open circles is the best-fitting model described by Equation A2. The same line was fitted to the closed circles by displacing it vertically. (B) Suppression depth as a function of test stimulus duration. Suppression depth was calculated by dividing sensitivity during suppression by that during dominance. The dashed line shows an absence of suppression, and each error bar indicates the $95 \%$ confidence interval.

between perceptual status (dominance or suppression) and azimuth was not significant $[F(15,64)=0.31, p=$ .993], indicating that suppression depth does not depend on azimuth. As a further test, we also performed a trend analysis on the suppression depths, using orthogonal polynomials up to order 5 . The polynomial of most interest is of order 4 , since it could show up dips or peaks separated in azimuth by $180^{\circ}$. This analysis did not prove significant $[F(1,32)=1.22, p=.28]$, nor did the analyses using polynomials of other orders $(p>.26)$. We therefore conclude that suppression depth is independent of the chromatic content of the test stimulus and, in particular, that chromatic channels coding for the red-green axis are no more heavily suppressed than are luminance channels.

\section{Experiment 2: Temporal \\ Selectivity of Suppression}

The question in the next experiment was whether suppression strength depends on test stimulus duration. The temporal sequence and placement of test stimuli were the same as those in the previous experiment and are shown in Figure 1A. The test stimulus differed from the background in luminance, but not in chromaticity. The independent variable in this experiment was test stimulus duration.
The results for 3 subjects are given in Figure 3A. The open circles show contrast sensitivity during the dominance phase of rivalry. Sensitivity increases with stimulus duration, as is required by the Bunsen-RoscoeBloch Law. The result of interest here is the sensitivity obtained during the suppression phase of rivalry and its relationship to the sensitivity during dominance. This relationship is brought out more clearly in panel B of the figure, which shows the sensitivity during suppression divided by that during dominance. There appears to be no consistent variation of suppression depth with stimulus duration. An ANOVA on the pooled sensitivities from the 3 subjects showed that the interaction between perceptual status (dominance or suppression) and duration did not account for a significant fraction of the variance $[F(7,32)=$ $0.098, p=.998]$. A second ANOVA was also performed to see if there was any trend in suppression depth with increasing duration. Orthogonal polynomials up to order 5 were fitted to the data, but all failed to reach significance $(p>.49)$. It is concluded that suppression strength does not vary systematically with the brevity of the stimulus.

Fox and Check (1972) also measured the temporal selectivity of suppression. They presented test stimuli of constant duration at one of three times during the interval for which a specific eye's conditioning stimulus was sup- 
Time Left eye view Right eye view

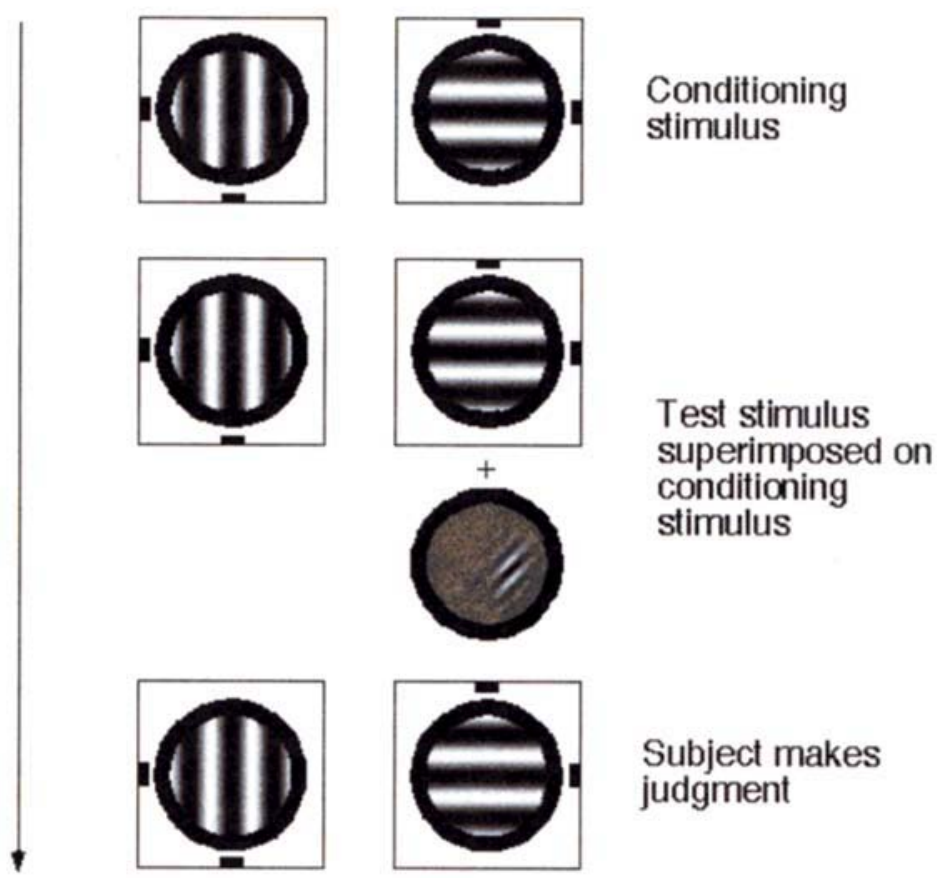

Figure 4. Stimulus used to test spatial vision during binocular rivalry. The conditioning stimulus was a sinusoidal grating for both eyes, with the left vertical and the right horizontal. Both gratings had a spatial frequency of $3 \mathrm{cycles}^{-1} \mathrm{deg}^{-1}$ and a contrast of $50 \%$. The test stimulus was a Gabor patch centered over either the left or the right half of the right eye's grating; only the latter case is shown. The subject was required to signal the test stimulus' location. The Gabor patch had a standard deviation in the horizontal direction equal to 0.24 times the fusion circle's radius and 0.35 times the radius in the vertical direction. The patch was oriented at $45^{\circ}$ to the horizontal, with a trough at its center. Test contrast was calculated relative to the mean luminance of the conditioning stimulus on which it was superimposed. The timing of the test stimulus is shown by the sequence from the top to the bottom of the figure.

pressed. They found that the fraction of correctly recognized test stimuli did not differ significantly between the early, middle, and late stages of a suppression phase. Our result is complementary to theirs, in that we find no selectivity for stimulus durations substantially shorter than those they used and in the lack of any selective effect on contrast sensitivity.

\section{Experiment 3: Spatial Selectivity of Suppression}

The aim in our third experiment was to determine whether binocular rivalry suppression produces a sensitivity loss that depends on the spatial patterning of the test stimulus. The stimulus used is shown in Figure 4. The conditioning stimulus consisted of a vertical grating to the left eye and a horizontal grating to the right, both gratings being 3 cycles $\mathrm{deg}^{-1}$ sinusoids. The test stimulus was a Gabor patch superimposed on either the left or the right half of the right eye grating. Superimposition was achieved by presenting the conditioning stimulus and the test stimulus on alternate video frames. The frame rate was $120 \mathrm{~Hz}$, so that both conditioning and test stimuli had a frame rate of $60 \mathrm{~Hz}$, with no apparent flicker. Test spatial frequency was set at one of six values between 1 and 10 cycles deg $^{-1}$. The subject triggered the test when the right eye's conditioning stimulus was either dominant or suppressed and then signaled the apparent location of the test.

Results are shown for 3 subjects in Figure 5A. Contrast sensitivity is plotted against test stimulus spatial frequency for both the dominance and the suppression phases of binocular rivalry. The data span only one decade of spatial frequency, because suppressed contrast sensitivity was too low to measure at higher spatial frequencies. The sensitivities collected during the dominance phase show the familiar band-pass function of spatial frequency (Robson, 1966). Panel B of the figure shows the loss of sensitivity owing to suppression: The ordinates in this case give the sensitivity during suppression divided by that during dominance. There seems to be no consistent trend in the depth of suppression with spatial frequency. We tested the hypothesis that suppression depth is independent of spatial frequency with an ANOVA. For the pooled 


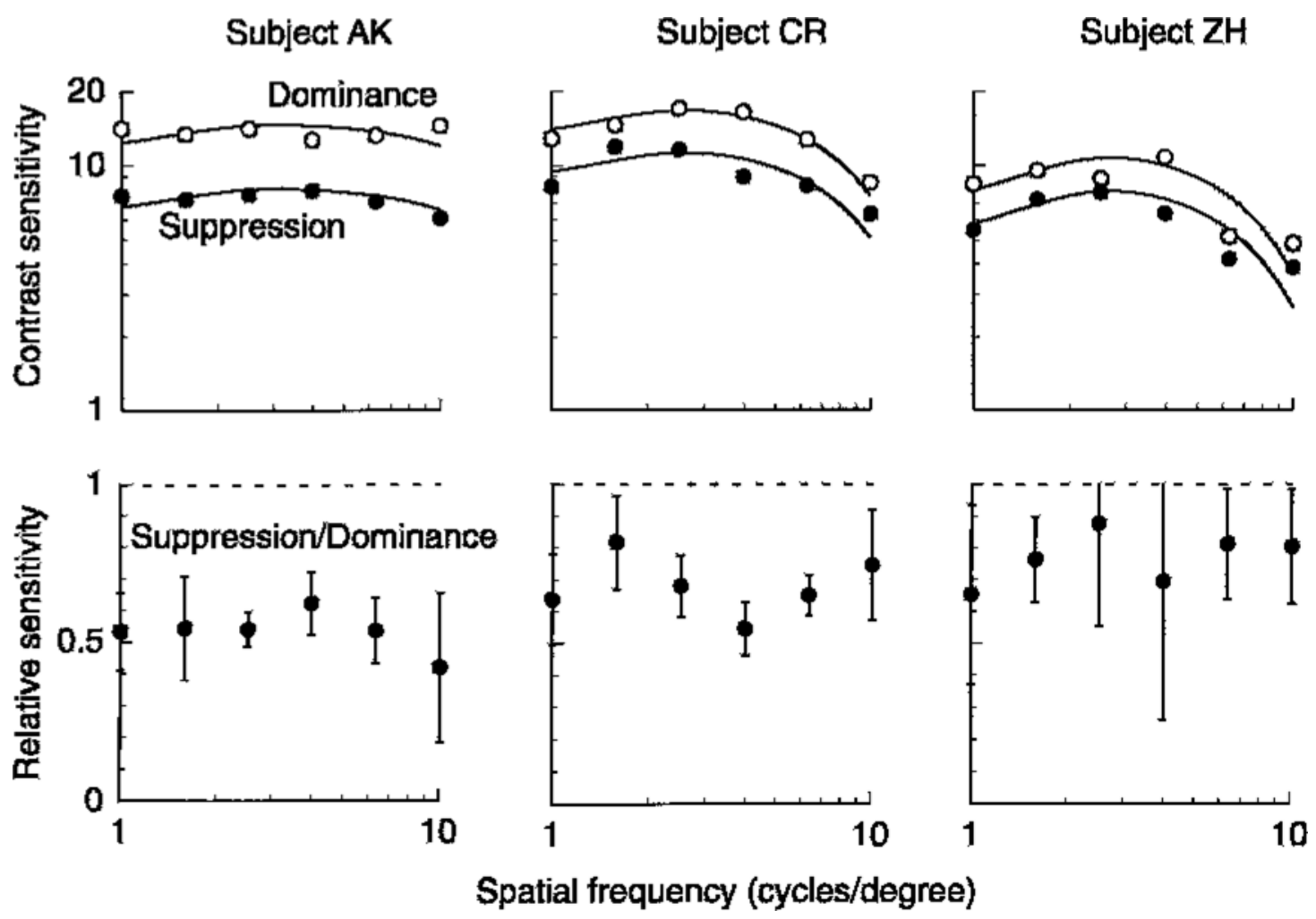

Figure 5. The effect of binocular rivalry suppression on the spatial frequency response. (A) Contrast sensitivity to a flashed grating as a function of the grating's spatial frequency. Results from 3 subjects are given. Sensitivity during the dominance and suppression phases of binocular rivalry is represented by open and filled circles, respectively. The line through the open circles gives the best-fitting difference-of-Gaussians model (Equation A3). The same model was displaced downward to fit the filled circles. (B) Suppression depth for the same subjects. Suppression depth was calculated by dividing the sensitivity during suppression with that during dominance. The dashed line indicates an absence of suppression, and each error bar indicates the $95 \%$ confidence interval.

sensitivities from the 3 subjects, the interaction term between perceptual status (dominance or suppression) and spatial frequency was not significant $[F(5,24)=0.065$, $p=.997]$. A trend analysis on suppression depth using orthogonal polynomials up to order 5 also failed to show significance $(p>.32)$. We conclude that suppression depth does not vary systematically with spatial frequency and that suppression is therefore not selective for the spatial frequency of the test stimulus.

\section{Experiment 4: Eye Suppression or Stimulus Suppression?}

Experiments 1-3 provide no evidence that binocular rivalry suppression is selective for specific test stimuli. The results thus far are therefore consistent with eye suppression: Suppression indiscriminately reduces the visibility of all stimuli to a given eye. Previous work has shown, however, that suppression has a component, stimulus suppression, that is not limited to a single monocular channel but is targeted at a stimulus characteristic, such as grating orientation (Logothetis et al., 1996) or chromaticity (Kovács et al., 1996). Our last experiment was therefore designed to test whether the suppression loss we measured was due to eye suppression or stimulus suppression. The distinguishing aspect of this experiment was that some of the test stimuli used were much like one or the other monocular conditioning stimulus. The stimulus was the same as that shown in Figure 4, except that here the test stimulus had a spatial frequency equal to that of the conditioning stimulus ( 3 cycles $\mathrm{deg}^{-1}$ ), an orientation that took one of seven values between, and including, horizontal and vertical, and a spatial phase that varied randomly from trial to trial (so that subjects could not base their judgments on luminance changes at a single location).

The results are shown in Figure 6A for 3 subjects. The open circles show the contrast sensitivity obtained when the conditioning stimulus to the tested eye is dominant. Sensitivity is lowest when the test is aligned with the conditioning stimulus on which it is superimposed and increases as the test is tilted away from the conditioning stimulus. This upward trend is presumably due to a release from monocular masking (Phillips \& Wilson, 1984). There is also a downturn in sensitivity at an orientation of $90^{\circ}$, where the test is aligned with the conditioning stimulus in the untested eye, an effect that is probably due to interocular masking (Legge, 1979).

The filled circles in Figure 6A give the contrast sensitivity when the conditioning stimulus to the tested eye is 
A

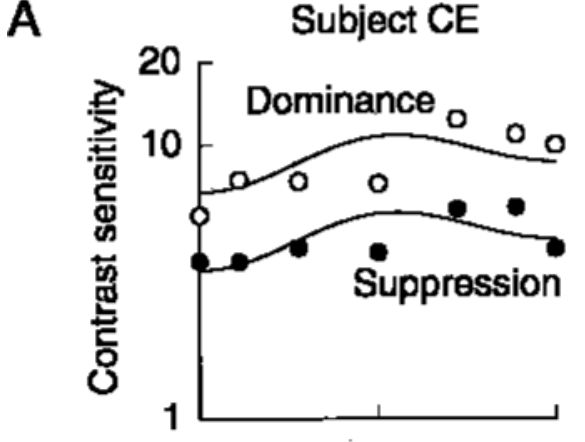

B

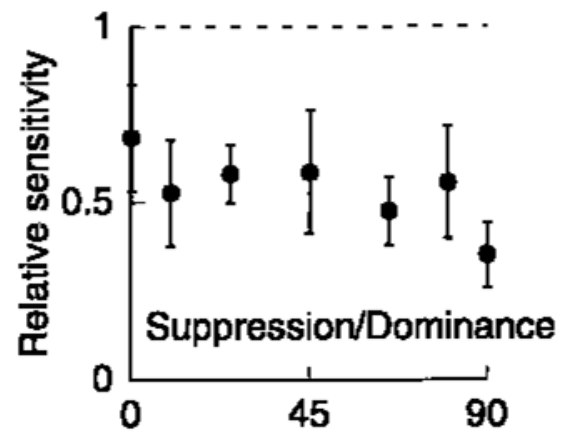

Subject CR
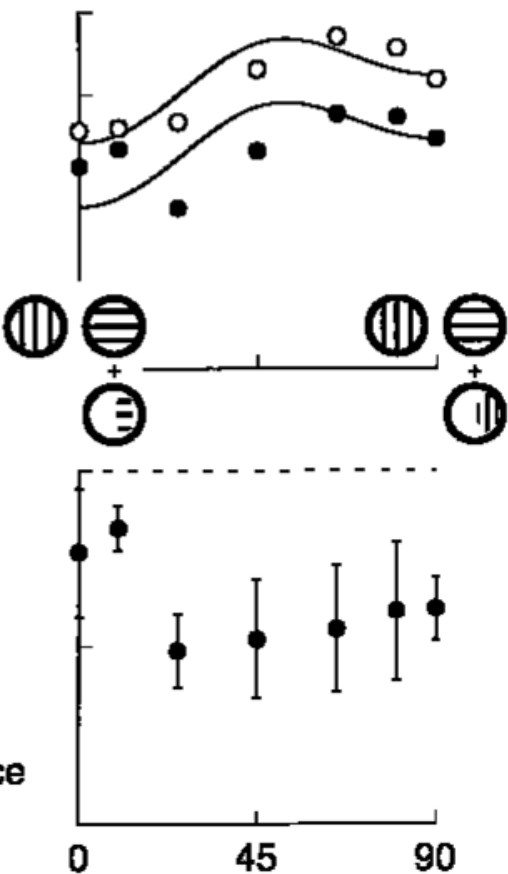

Subject FL
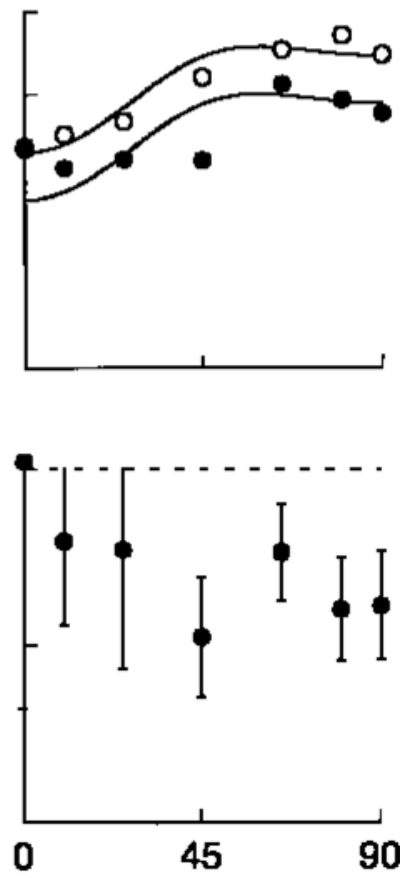

\section{Orientation (degrees)}

Figure 6. Testing for eye or stimulus suppression. (A) Contrast sensitivity for 3 subjects. Open and filled circles give sensitivity when the conditioning stimulus before the tested eye was dominant or suppressed, respectively. The line through the open circles is the best-fitting masking model (Equation A4). The same model was displaced downward to fit the filled circles. Figurines on the horizontal axis show test orientation relative to conditioning stimulus orientation. (B) Suppression depth, obtained by dividing sensitivity during suppression by that during dominance. The dashed line indicates equal sensitivities for dominance and suppression, and each error bar indicates the $95 \%$ confidence interval.

suppressed, and the values in panel B of the figure show the sensitivity during suppression divided by that during dominance. An ANOVA on the pooled sensitivities from the 3 subjects showed that the interaction term between perceptual status (dominance or suppression) and orientation did not reach significance $[F(6,28)=0.82, p=.57]$. A trend analysis, however, produced a different result. Using orthogonal polynomials up to order 5 , the linear term reached significance $[F(1,14)=7.86, p=.014]$. This result, seen in Figure 6B as a downward trend in relative sensitivity with increasing orientation, is not very robust. Removing one point, that of subject F.L. at an orientation of $0^{\circ}$, shifts the $F$-value to the edge of significance $[F(1,13)=4.67, p=.05]$. We conclude, therefore, that suppression of the conditioning stimulus to the tested eye produces a loss of sensitivity that depends weakly on test orientation.

The results in Figure 6 closely match the expectations of the eye suppression hypothesis. Assume, on the contrary, that the stimulus suppression hypothesis is true. What do we expect then? Consider the data obtained with a test orientation of $90^{\circ}$. The open circle at this orientation is obtained when the horizontal conditioning stimulus is dominant and the vertical conditioning stimulus is suppressed. The test stimulus has the same orientation as the suppressed conditioning stimulus and, according to the hypothesis, should be suppressed. The open circle should therefore lie below its filled counterpart, resulting in a suppression/dominance ratio greater than 1 . A $t$ test shows that the ratio is less than 1 at a test orientation of $90^{\circ}$ [ratio $=0.52 ; t(11)=10.0, p<.001]$, forcing the rejection of the stimulus suppression hypothesis for the conditions in which our experiment was performed.

\section{DISCUSSION}

\section{Selectivity of Suppression}

Our results are in agreement with previously published reports (Blake \& Fox, 1974b; Blake et al., 1980; Fox \& Check, 1968; Wales \& Fox, 1970) about the selectivity of binocular rivalry suppression: Suppression depth varies little or not at all with the type of stimulus used to test it. This agreement extends to the results of E. L. Smith et al. (1982). Their Figure 1 shows that spectral sensitivity during suppression is less than that during dominance and that there is no clear dependence of suppression depth on stimulus wavelength at wavelengths above $480 \mathrm{~nm}$. Wavelengths below $480 \mathrm{~nm}$ have little influence on M- and L-cone contrast, so our data relate largely to the upper end of the wavelength range. The pres- 
ent results and those of Smith et al. therefore agree that suppression is nonselective when the test stimulus is varied in its medium and high wavelength content.

E. L. Smith et al. (1982) interpreted their data to mean that suppression acts more strongly on chromatic channels than on luminance channels. The results obtained in the present paper indicate that this interpretation can be made more precise. Sperling and Harwerth (1971) showed that signals in two chromatic channels (L-M and $\mathrm{M}-\mathrm{L}$ ) depend on a difference between $\mathrm{L}$ - and $\mathrm{M}$-cone responses and that signals in a third channel derive from S-cones. We have shown that channels signaling a difference between $\mathrm{L}-$ and $\mathrm{M}$-cone responses are suppressed no more strongly than luminance channels. The large suppression depth that E. L. Smith et al. demonstrated at low wavelengths is therefore likely to be due to a specific effect on the channel in which S-cones play a major role.

In the spatial domain, three previous studies (Blake \& Fox, 1974b; O'Shea \& Crassini, 1981; Walker \& Powell, 1979) have investigated whether a change in the spatial frequency or orientation of a suppressed stimulus is sufficient to break suppression. All three studies used a replacement procedure to introduce the stimulus change: The suppressed conditioning stimulus, a square-wave grating, was replaced by another stimulus differing only in spatial frequency or orientation. Such a procedure introduces sudden changes in luminance across the stimulus at places where dark bars are replaced with light and light with dark. The suppression-breaking power of stimulus replacement presumably depends on the area of these luminance changes. The difficulty with the replacement procedure, therefore, is that breaking suppression probably depends more on the difference between the original and the replacement conditioning stimuli and less on the spatial form of the individual stimuli. It is not surprising, therefore, that the three studies produced differing results. Blake and Fox (1974b) who used conditioning stimuli of low contrast (9\% or less), found no break in suppression when frequency or orientation changes, whereas Walker and Powell and O'Shea and Crassini, using contrasts of at least $70 \%$, found clear breaks in suppression.

One way of removing the confusion between luminance and spatial change is to measure the threshold of a test stimulus presented during the suppression phase. This is the approach we have taken, and it is also the method used by O'Shea and Crassini (1981) in their second experiment. They still used a replacement procedure, but the duration of the replacement grating was adjusted so that the subjects indicated its orientation with a specified accuracy. Rivalry was induced with orthogonal gratings, and one grating was briefly altered in orientation while either suppressed or dominant. They found that thresholds during suppression were much larger than those during dominance when the orientation change was less than $20^{\circ}$ or greater than $70^{\circ}$ but that thresholds differed little in the $20^{\circ}-70^{\circ}$ range.

This disagrees with our finding of a nearly constant threshold increase for all test stimulus orientations. The reason for the disagreement is not clear but may be due to different methodology. For instance, whereas we used contrast as our threshold measure, O'Shea and Crassini (1981) used duration. The large threshold increases they found in some cases may have been due to durations near or exceeding the integration time for stimulus detection. It is useful to note, though, that their results and ours agree in one important respect. Suppression is present for an orientation change of, or close to, $90^{\circ}$, where the suppressed stimulus is aligned, or nearly so, with the dominant stimulus. The continued suppression in this case argues strongly for eye suppression, as opposed to stimulus suppression. O'Shea and Crassini's study and our study are therefore in agreement on this point.

The one case in which we found any selectivity in the suppression process was in Experiment 4: There was deeper suppression when test stimulus orientation was set near that of the dominant conditioning stimulus. This trend is in the opposite direction to that expected from the stimulus suppression hypothesis, in which a test aligned with the dominant conditioning stimulus would lead to enhanced sensitivity. The selectivity of suppression seen in Figure 6 is therefore further evidence against stimulus suppression.

\section{Suppression Depth}

Several previous studies have measured the depth of suppression by inducing binocular rivalry, delivering a test stimulus to one eye during its dominance and suppression phases and comparing test stimulus sensitivity between the two phases. Wales and Fox (1970) used such a method, and their result is frequently quoted. They found that sensitivity for test stimulus detection during suppression was on the order of $0.5 \mathrm{log}$ units less than, and therefore $32 \%$ of the value of, that during dominance. Their result, however, was obtained by extrapolation along a psychometric function over a substantial fraction of the response range. More reliable measurements were made by Makous and Sanders (1978) and Blake and Camisa (1979). From data in those publications (Figures 14.2 and 6, respectively), it can be estimated that sensitivity during suppression drops to about $60 \%$ of that during dominance. Our results match well with those of Makous and Sanders and those of Blake and Camisa. Sensitivity during suppression averaged $60 \%, 65 \%, 66 \%$, and $63 \%$ of that during dominance in Experiments 1-4, respectively.

\section{Perceptual Loss in Suppression}

There are two findings in our work that are puzzling. First, the depth of suppression we have measured is surprisingly small when compared with the perceptual loss during suppression. The sensitivity to a test stimulus during suppression drops to an average of $64 \%$ of that during dominance, but the suppressed conditioning stimulus disappears completely from sight. In perceptual terms, the contrast sensitivity for the conditioning stimulus must drop by an order of magnitude or two during suppression. Why is there such a discrepancy between the small drop in contrast sensitivity for a flashed test and the large perceptual loss during suppression? A second puzzle in our 
findings is the rejection of the stimulus suppression hypothesis in Experiment 4. Recent psychophysical results indicate that the perceptual loss during binocular rivalry is specific for color (Kovács et al., 1996) or, in some circumstances, grating orientation (Lee \& Blake, 1999; Logothetis et al., 1996). We find, however, that suppression depth is much the same whether the test stimulus is similar or dissimilar to the suppressed conditioning stimulus.

A hypothesis capable of resolving both of these puzzles is that the losses in test detection and conditioning stimulus perception are mediated by different sites in the visual system. Under this hypothesis, losses in test detection are assumed to occur within the primary visual cortex, and perceptual losses are confined to the higher visual cortex. The evidence for this idea comes partly from studies of the influence of binocular rivalry on visual aftereffects. One aftereffect studied in this way is the apparent reduction in grating contrast that occurs after a suprathreshold grating has been viewed for a prolonged period. Another such aftereffect occurs when a stationary grating is viewed after adaptation to a translating grating: The stationary grating appears to move in the opposite direction. Binocular rivalry rendering the adapting stimulus invisible for some of its presentation time does not reduce the strength of these aftereffects (Blake \& Fox, 1974a; Lehmkuhle \& Fox, 1975). Because these aftereffects are assumed to arise in the primary visual cortex, the corollary is that binocular rivalry suppression is targeted at sites beyond the primary area.

By contrast, binocular rivalry does reduce motion aftereffects induced with a rotating spiral (Wiesenfelder \& Blake, 1990) or drifting plaid (van der Zwan, Wenderoth, $\&$ Alais, 1993). These aftereffects are assumed to arise beyond the primary visual cortex, since cells specifically responsive to such forms of motion are found in cortical areas MST (Graziano, Andersen, \& Snowden, 1994) and MT (Movshon, Adelson, Gizzi, \& Newsome, 1985), respectively, but not in the primary cortex. These studies together provide substantial evidence that perceptual losses owing to rivalry suppression arise at cortical sites beyond the primary visual area. Spatial and temporal resolutions, on the other hand, are at a maximum in the primary visual cortex and decline in higher order areas owing to convergence (Barlow, 1981). It is highly likely, therefore, that detection of the test stimulus takes place in the primary visual cortex.

There is also physiological evidence supporting the suggestion that several cortical sites are involved in rivalry. First, Sengpiel, Blakemore, and Harrad (1995) studied lateral geniculate cells and monocular cells in the primary visual cortex of the anesthetized cat. They found that the response to a drifting grating presented to the dominant eye was inhibited by a grating presented to the fellow eye but that the inhibition was unaffected by the relative orientation of the two gratings. Furthermore, the loss of response was clustered around the $50 \%$ mark. Both of these findings show clear parallels with the results of our Experiment 4. Sengpiel et al. also studied binocular cells in the primary visual cortex. These cells were also inhibited by binocular stimulation, but only when the two gratings differed in orientation by more than about $20^{\circ}$. Second, Sheinberg and Logothetis (1997) presented macaque monkeys with binocularly rival stimuli and trained the monkeys to indicate continuously which of the two stimuli was perceived. Activity of cells in the inferior temporal cortex was recorded during the behavioral task. There was a good correlation between the activity of many of the cells and the behavioral responses, indicating that cells at this cortical level may have a major role in the perceptions underlying rivalry.

The hypothesis that test detection and conditioning stimulus perception occur at differing sites explains our two puzzles as follows. First, sensitivity losses in suppression are low because test detection occurs before signals reach the area at which binocular rivalry suppression is targeted, and perceptual losses are high because they are mediated by an area strongly affected by such suppression. Second, sensitivity losses are better explained by eye suppression than by stimulus suppression, because they occur in the primary visual cortex, where a significant fraction of the cells are monocular or dominated by one eye.

Figure 7 provides a model consistent with such data. It is assumed, in common with previous models (Lehky, 1988; Sugie, 1982), that there is mutual inhibition between left- and right-eye-dominated cells. Consistent with the physiological evidence (Sengpiel et al., 1995), it is assumed that this inhibition does not depend on the similarity, or otherwise, of the stimuli to the two eyes. It is also assumed that there is inhibition between binocular cell populations responding to opposed stimulus features. For the purposes of illustration, the figure shows inhibition between only two groups of cells, those tuned to vertical orientations and those tuned to horizontal. Unlike previous models, it is assumed here that the inhibition occurs in both the primary visual cortex and the higher cortical levels. The suppressed cells at the higher levels in this model receive a smaller excitatory input than do the suppressed cells at the primary level. Inhibition at successive levels therefore results in amplification of suppression depth. If the sensitivity for detection of a test stimulus is mediated at the primary visual cortex and the percept at the higher level, the model can explain the marked difference between detection sensitivity and perceptual loss.

The model assumes that there is interocular suppression in the primary visual cortex and that there is a progressive increase in suppression at each successive level of the visual cortex. This, in turn, predicts that aftereffects that arise in the primary cortex should be at least marginally reduced by binocular rivalry during adaptation. A small reduction of such aftereffects is consistent with the fact that, although early reports found no significant reduction of aftereffects thought to arise in the primary visual cortex (Blake \& Fox, 1974a; Lehmkuhle \& Fox, 1975), a more recent report does find a significant reduction (Lehky \& Blake, 1991). Also consistent with increasing rivalry suppression in the higher visual cortex is the finding that af- 


\title{
Visual stimulus
}

\author{
Activity in primary \\ visual cortex
}

Activity in higher

visual cortex

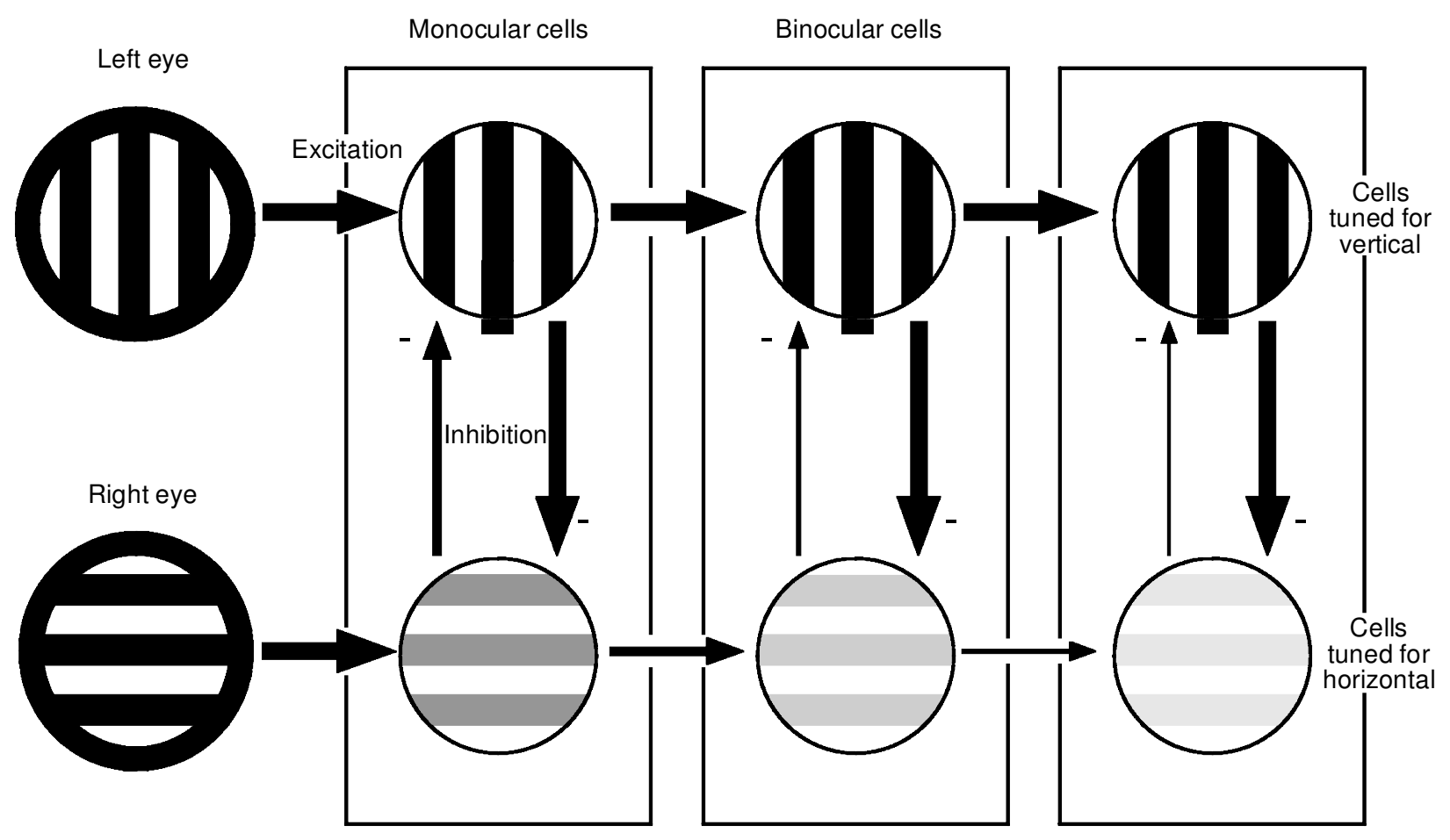

Figure 7. A distributed model for binocular rivalry suppression. The leftmost column indicates the rivalry-inducing stimulus. The first box represents the activity of monocular cells in the primary visual cortex, and the remaining boxes show activity at locations more advanced along the visual pathway. Gratings in the boxes indicate the activity of cells, with higher activities shown by higher contrasts. The arrows show signals: Thicker arrows indicate stronger signals, horizontal arrows show excitation, and vertical arrows inhibition. The figure represents a snapshot of activity during a period in which the right eye's stimulus is suppressed. Activity in horizontally tuned cells is less in the higher visual cortex than in the primary cortex because they receive less excitation. This model explains the relatively large loss in perception, compared with that in sensitivity, so long as perception of the background stimulus is mediated by the higher visual cortex and sensitivity to the test stimulus is mediated by the primary cortex. If inhibition between monocular cells is assumed to be independent of the similarity, or lack of it, between stimuli to the two eyes, the model also explains the eye suppression we found.

tereffects that are thought to be encoded by such areas are markedly reduced by rivalry during adaptation (van der Zwan et al., 1993; Wiesenfelder \& Blake, 1990).

\section{REFERENCES}

BARLOw, H. B. (1981). Critical limiting factors in the design of the eye and visual cortex. Proceedings of the Royal Society of London: Series $B, \mathbf{2 1 2}, 1-34$.

BlAKe, R., \& CAMISA, J. (1979). On the inhibitory nature of binocular rivalry suppression. Journal of Experimental Psychology, 5, 315-323.

BlAKe, R., \& Fox, R. (1974a). Adaptation to invisible gratings and the site of binocular rivalry suppression. Nature, 249, 488-490.

BLAKE, R., \& FoX, R. (1974b). Binocular rivalry suppression: Insensitive to spatial frequency and orientation change. Vision Research, 14, 687-692.

Blake, R., O'Shea, R, \& Mueller, T. J. (1992). Spatial zones of binocular rivalry in central and peripheral vision. Visual Neuroscience, 8, 469-478.

Blake, R, Westendorf, D. H., \& Overton, R. (1980). What is suppressed during binocular rivalry? Perception, 9, 223-231.

Chaparro, A., Stromeyer, C. F., Huang, E. P., Kronauer, R. E., \& ESKEW, R. T., JR. (1993). Colour is what the eye sees best. Nature, 361, 348-350.
Cole, G. R., Hine, T., \& McIlhagga, W. (1993). Detection mechanisms in L-, M-, and S-cone contrast space. Journal of the Optical Society of America A, 10, 38-51.

DuTour, E.-F. (1760). Discussion d'une question d'optique. L'Académie des Sciences. Mémoirs de Mathématique et de Physique Présentés par Divers Savants, 3, 514-530. (See psy.otago.ac.nz:800/r_oshea/ dutour60.html for a translation by R. P. O'Shea.)

Enroth-Cugell, C., \& Robson, J. G. (1966). The contrast sensitivity of retinal ganglion cells of the cat. Journal of Physiology, 187, 517552.

Fox, R., \& CHeck, R. (1968). Detection of motion during binocular rivalry suppression. Journal of Experimental Psychology, 78, 388-395.

Fox, R., \& CHECK, R. (1972). Independence between binocular rivalry suppression duration and magnitude of suppression. Journal of Experimental Psychology, 93, 283-289.

Graziano, M. S. A., Andersen, R. A., \& Snowden, R. J. (1994). Tuning of MST neurons to spiral motions. Journal of Neuroscience, 14, 54-67.

Kovács, I., Papathomas, T. V., Yang, M., \& Fehér, A. (1996). When the brain changes its mind: Interocular grouping during binocular rivalry. Proceedings of the National Academy of Sciences, 93, 15508-15511. LEE, S., \& BLAKE, R. (1999). Rival ideas about binocular rivalry. Vision Research, 39, 1447-1454.

LEGGE, G. E. (1979). Spatial frequency masking in human vision: Binocular interactions. Journal of the Optical Society of America, 69, 838-847. 
LEHKY, S. R. (1988). An astable multivibrator model of binocular rivalry. Perception, 17, 215-228.

LEHKY, S. R., \& BLAKE, R. (1991). Organization of binocular pathways: Modeling and data related to rivalry. Neural Computation, 3, 44-53.

LeHmKuhle, S. W., \& Fox, R. (1975). Effect of binocular rivalry suppression on the motion aftereffect. Vision Research, 15, 855-859.

Logothetis, N. K., Leopold, D. A., \& Sheinberg,D. L. (1996). What is rivalling during binocular rivalry? Nature, 380, 621-624.

MaKous, W., \& SANDERS, R. K. (1978). Suppression interactions between fused patterns. In A. C. Armington, J. Krauskopf, \& B. R. Wooten (Eds.), Visual psychophysics and physiology (pp. 167-179). New York: Academic Press.

Movshon, J. A., Adelson, E. H., Gizzi, M. S., \& Newsome, W. T. (1985). The analysis of moving visual patterns. In C. Chagas, R. Gattass, \& C. Gross (Eds.), Pattern recognition mechanisms (pp. 117-151). Berlin: Springer-Verlag.

Nguyen, V. A., \& Freeman, A. W. (1997). Depth and selectivity of interocular suppression [Abstract]. Proceedings of the Australian Neuroscience Society, $\mathbf{8}, 164$.

Nguyen, V. A., Freeman, A. W., Wenderoth, P. M., \& Heard, R. C. (1998). Interocular suppression: Large perceptual loss, small sensitivity loss. In N. Kasabov, R. Kozma, K. Ko, R. O’Shea, G. Coghill, \& T. Gedeon (Eds.), Progress in connectionist-based information systems (Vol. 1, pp. 51-54). Singapore: Springer-Verlag.

OoI, T. L., \& Loop, M. S. (1994). Visual suppression and its effect upon color and luminance sensitivity. Vision Research, 34, 2997-3003.

O'Shea, R. P., \& Crassini, B. (1981). The sensitivity of binocular rivalry suppression to changes in orientation assessed by reaction-time and forced-choice techniques. Perception, 10, 283-293.

Phillips, G. C., \& Wilson, H. R. (1984). Orientation bandwidths of spatial mechanisms measured by masking. Journal of the Optical Society of America A, 1, 226-232.

Robson, J. G. (1966). Spatial and temporal contrast-sensitivity func- tions of the visual system. Journal of the Optical Society of America, 56, 1141-1142.

Sengpiel, F., Blakemore, C., \& Harrad, R (1995). Interocular suppression in the primary visual cortex: A possible neural basis of binocular rivalry. Vision Research, 35, 179-195.

Sheinberg, D. L., \& Logothetis, N. K. (1997). The role of temporal cortical areas in perceptual organization. Proceedings of the National Academy of Sciences, 94, 3408-3413.

Smith, E. L., Levi, D. M., Harwerth, R. S., \& White, J. M. (1982). Color vision is altered during the suppression phase of binocular rivalry. Science, 218, 802-804.

Smith, V. C., \& Pokorny, J. (1975). Spectral sensitivity of the foveal cone photopigments between 400 and $500 \mathrm{~nm}$. Vision Research, 15, 161-171.

Sperling, H. G., \& HARWERTh, R. S. (1971). Red-green cone interactions in the increment-threshold spectral sensitivity of primates. Science, 172, 180-184.

SugIE, N. (1982). Neural models of brightness perception and retinal rivalry in binocular vision. Biological Cybernetics, 43, 13-21.

van Der Zwan, R., Wenderoth, P., \& Alais, D. (1993). Reduction of a pattern-induced motion aftereffect by binocular rivalry suggests the involvement of extrastriate mechanisms. Visual Neuroscience, 10, 703-709.

WALES, R., \& FoX, R. (1970). Increment detection thresholds during binocular rivalry suppression. Perception \& Psychophysics, 8, 90-94.

WALKer, P., \& Powell, D. J. (1979). The sensitivity of binocular rivalry to changes in the nondominant stimulus. Vision Research, 19, 247-249.

Weibull, W. A. (1951). A statistical distribution function of wide applicability. Journal of Applied Mechanics, 18, 292-297.

Wiesenfelder, H., \& BlAKe, R. (1990). The neural site of binocular rivalry relative to the analysis of motion in the human visual system. Journal of Neuroscience, 10, 3880-3888.

\section{APPENDIX}

Four models were fitted to the experimental data. This appendix defines the models and describes the fitting method.

\section{Experiment 1: Chromatic Model}

The first model, which appears in Figure 2, is a probability summation model for contrast threshold as a function of L- and M-cone contrast. This was derived from Equation A4 in Cole et al. (1993):

$$
\begin{aligned}
\mathrm{P}_{\text {correct }} & =0.79 \\
& =1-0.5 \exp \left\{-_{i=1} \Sigma^{2}\left[l_{i} c \cos (\Theta)+m_{i} c \sin (\Theta)\right]^{4}\right\},
\end{aligned}
$$

where $l_{i}, m_{i}=$ weighting of the L- and M-cone contrasts in activity of the $\mathrm{L}+\mathrm{M}(i=1)$ and the $\mathrm{L}-\mathrm{M}(i=2)$ channels, $c=$ stimulus contrast, and $\Theta=$ azimuth (deg).

Solving for threshold,

$$
c=\left(-\{\ln [2(1-0.79)]\} /{ }_{i=1} \sum^{2}\left[l_{i} \cos (\Theta)+m_{i} \sin (\Theta)\right]^{4}\right)^{1 / 4} .
$$

\section{Experiment 2: Temporal Model}

The second model, seen in Figure 3, is a model for sensitivity as a function of test stimulus duration:

$$
\text { sensitivity }=\left(S_{\max } d / d_{\mathrm{c}}\right) / \sqrt{ }\left[\left(d / d_{\mathrm{c}}\right)^{2}+1\right],
$$

where $S_{\max }=$ maximum sensitivity, $d=$ test stimulus duration ( $\mathrm{sec}$ ), and $d_{\mathrm{c}}=$ corner duration ( $\mathrm{sec}$ ), at which asymptotes meet.

\section{Experiment 3: Spatial Model}

The third model, seen in Figure 5, is a difference-of-Gaussians model for sensitivity to a grating as a function of the grating's spatial frequency (Enroth-Cugell \& Robson, 1966):

$$
\text { sensitivity }=S_{\mathrm{c}} \exp \left[-\left(\pi r_{\mathrm{c}} u\right)^{2}\right]-S_{\mathrm{s}} \exp \left[-\left(\pi r_{\mathrm{s}} u\right)^{2}\right],
$$

where $S_{\mathrm{c}}, S_{\mathrm{s}}=$ sensitivities of the center and surround mechanisms, $r_{\mathrm{c}}, r_{\mathrm{s}}=$ radii (in degrees) of the center and surround mechanisms, and $u=$ grating spatial frequency (cycles deg ${ }^{-1}$ ).

\section{Experiment 4: Orientation Model}

The last model assumes that sensitivity to a Gabor test stimulus is reduced by both monocular and interocularmasking. The attenuation owing to masking depends on a Gaussian function of the orientation difference between the test and the mask:

$$
\begin{aligned}
\text { sensitivity }= & S_{0}\left\{1+k_{\mathrm{m}} e^{-0.69\left[\Theta / \Theta_{\mathrm{m}}\right]^{2}}\right\}^{-1} \\
& \left\{1+k_{\mathrm{i}} e^{-0.69\left[(90-\Theta) / \Theta_{i}\right]^{2}}\right\}^{-1},
\end{aligned}
$$

where $S_{0}=$ maximum sensitivity, $\Theta=$ orientation (deg) relative to horizontal, $\Theta_{\mathrm{m}}, \Theta_{\mathrm{i}}=$ half-width at half-height (in degrees) of sensitivity loss owing to monocular and interocular masking, 


\section{APPENDIX (Continued)}

respectively, and $k_{\mathrm{m}}, k_{\mathrm{i}}=$ depth of monocular and interocular masking, respectively.

Phillips and Wilson (1984) found a masking function with a half-width at half-height of $25^{\circ}$ for 3 cycles $\mathrm{deg}^{-1}$ gratings. Both $\Theta_{m}$ and $\Theta_{i}$ were set equal to this value.

\section{Model Fitting}

All four models were fitted to the experimental data by minimizing the sum of squared errors between the model and the observations. The Solver function in Microsoft Excel was used to perform the minimization.

(Manuscript received April 21, 1998;

revision accepted for publication April 29, 2000.) 